\title{
Ground state sign-changing solutions for a class of double-phase problem in bounded domains
}

\author{
Gangling Hou' ${ }^{1}$ Bin $\mathrm{Ge}^{2,3^{*}}$ (D), Beilei Zhang ${ }^{2}$ and Liyan Wang ${ }^{3}$
}

\section{"Correspondence:}

gebin791025@hrbeu.edu.cn

${ }^{2}$ School of Mathematical Sciences,

Harbin Engineering University,

Harbin, P.R. China

${ }^{3}$ College of Automation, Harbin

Engineering University, Harbin, P.R.

China

Full list of author information is

available at the end of the article

\begin{abstract}
This paper is concerned with the following double-phase problem:

$$
\begin{cases}-\operatorname{div}\left(|\nabla u|^{p-2} \nabla u+a(x)|\nabla u|^{q-2} \nabla u\right)=f(x, u) & \text { in } \Omega, \\ u=0 & \text { on } \partial \Omega,\end{cases}
$$

where $N \geq 2$ and $1<p<q<N$. Assuming that the primitive of $f(x, u)$ is asymptotically $q$-linear as $|u| \rightarrow \infty$ and a weak version of Nehari-type monotonicity condition that the function $u \mapsto \frac{f(x, u)}{|u|^{q-1}}$ is nondecreasing on $(-\infty, 0) \cup(0, \infty)$ for a.e. $x \in \Omega$, we prove the existence of one ground state sign-changing solution via the constraint variational method and quantitative deformation lemma for the equation. Our results improve and generalize some results obtained by Liu and Dai (J. Differ. Equ. 265(9):4311-4334, 2018).
\end{abstract}

Keywords: Double-phase problem; Musielak-Orlicz space; Variational method; Ground state sign-changing solutions; Nehari manifold; Perturbation method

\section{Introduction and main results}

Differential equations and variational problems with double phase operator are a new and interesting topic. It arises from the nonlinear elasticity theory, strongly anisotropic materials, Lavrentiev's phenomenon, and so on (see [2-5]). The study on double-phase problems attracts more and more interest in recent years, and many results have been obtained [1, 6-10]. More precisely, the research is related to the energy functional

$$
u \mapsto \int_{\Omega}\left(|\nabla u|^{p}+a(x)|\nabla u|^{q}\right) d x
$$

where the integrand switches between two different elliptic behaviors. In [5], energies of the form (1) are used in the context of homogenization and elasticity, and the function $a$ drives the geometry of a composite of two different materials with hardening powers $p$ and $q$.

(c) The Author(s) 2020. This article is licensed under a Creative Commons Attribution 4.0 International License, which permits use sharing, adaptation, distribution and reproduction in any medium or format, as long as you give appropriate credit to the original author(s) and the source, provide a link to the Creative Commons licence, and indicate if changes were made. The images or other third party material in this article are included in the article's Creative Commons licence, unless indicated otherwise in a credit line to the material. If material is not included in the article's Creative Commons licence and your intended use is not permitted by statutory regulation or exceeds the permitted use, you will need to obtain permission directly from the copyright holder. To view a copy of this licence, visit http://creativecommons.org/licenses/by/4.0/. 
In this paper, we are concerned with the existence of sign-changing solutions of the double-phase problem

$$
\begin{cases}-\operatorname{div}\left(|\nabla u|^{p-2} \nabla u+a(x)|\nabla u|^{q-2} \nabla u\right)=f(x, u) & \text { in } \Omega, \\ u=0 & \text { on } \partial \Omega\end{cases}
$$

where $\Omega$ is a smooth bounded domain in $\mathbb{R}^{N}, N \geq 2,1<p<q<N$, and

$$
\frac{q}{p}<1+\frac{1}{N}, \quad a: \bar{\Omega} \mapsto[0,+\infty) \quad \text { is Lipschitz continuous, }
$$

and $f: \Omega \times \mathbb{R} \mapsto \mathbb{R}$ is a Carathéodory function satisfying the following assumptions:

$\left(h_{1}\right) f(x, t)=o\left(|t|^{p-2} t\right)$ as $t \rightarrow 0$ uniformly in $x \in \Omega$;

$\left(h_{2}\right)$ there exist $q<r<p^{*}$ and some positive constant $C$ such that

$$
|f(x, t)| \leq C\left(1+|t|^{r-1}\right)
$$

where $p^{*}=\frac{N p}{N-p}$ is the critical exponent.

(h) $\lim _{|t| \rightarrow+\infty} \frac{F(x, t)}{|t|^{q}}=+\infty$ uniformly in $x \in \Omega$, where $F(x, t)=\int_{0}^{t} f(x, s) d s$;

$\left(h_{4}\right)$ the function $t \mapsto \frac{f(x, t)}{|t|^{q-1}}$ is nondecreasing on $(-\infty, 0) \cup(0,+\infty)$ for a.e. $x \in \Omega$.

The solution of $(P)$ is understand in the weak sense, that is, $u \in W_{0}^{1, H}(\Omega)$ is a solution of $(P)$ if

$$
\begin{gathered}
\int_{\Omega}\left(|\nabla u|^{p-2} \nabla u \cdot \nabla v+a(x)|\nabla u|^{q-2} \nabla u \cdot \nabla v\right) d x \\
=\int_{\Omega} f(x, u) v d x, \quad \forall v \in W_{0}^{1, H}(\Omega)
\end{gathered}
$$

where $W_{0}^{1, H}(\Omega)$ will be defined in Sect. 2 .

Note that energy functional $\varphi$ associated with $(P)$ is defined by

$$
\varphi(u)=\int_{\Omega}\left(\frac{1}{p}|\nabla u|^{p}+\frac{a(x)}{q}|\nabla u|^{q}\right) d x-\int_{\Omega} F(x, u) d x .
$$

It is a well-known consequence of $\left(h_{1}\right)$ and $\left(h_{2}\right)$ that $\varphi \in C^{1}\left(W_{0}^{1, H}(\Omega), \mathbb{R}\right)$ and the critical points of $\varphi$ are weak solutions of $(P)$. Furthermore, if $u \in W_{0}^{1, H}(\Omega)$ is a solution of $(P)$ and $u^{ \pm} \neq 0$, then $u$ is a sign-changing solution of $(P)$, where

$$
u^{+}(x):=\max \{u(x), 0\} \quad \text { and } \quad u^{-}(x):=\min \{u(x), 0\} .
$$

To facilitate the narrative, we set

$$
\begin{aligned}
& \mathbb{M}_{0}:=\left\{u \in W_{0}^{1, H}(\Omega): u^{ \pm} \neq 0,\left\langle\varphi^{\prime}(u), u^{+}\right\rangle=\left\langle\varphi^{\prime}(u), u^{-}\right\rangle=0\right\} \\
& \mathbb{N}_{0}:=\left\{u \in W_{0}^{1, H}(\Omega): u \neq 0,\left\langle\varphi^{\prime}(u), u\right\rangle=0\right\},
\end{aligned}
$$

and put

$$
m_{0}:=\inf _{u \in \mathbb{M}_{0}} \varphi(u), \quad n_{0}:=\inf _{u \in \mathbb{N}_{0}} \varphi(u)
$$


Let us recall some previous results that led us to the present research. The first result is due to Perera and Squassina [6], who considered the following form of $(P)$ with the $q$ superlinear nonlinearity:

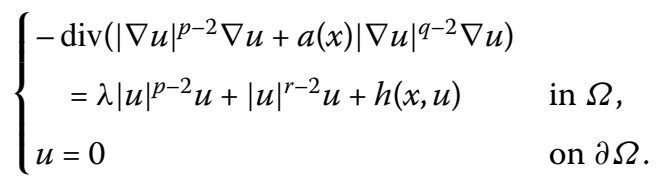

Applying the Morse theory, they proved that $\left(P_{1}\right)$ has a nontrivial solution by assuming that either

(T, $\lambda \notin\left\{\lambda_{k}\right\}_{k=1}^{\infty}$; or

$\left(T_{2}\right)$ for some $\delta>0, \frac{|t|^{r}}{r}+H(x, t) \leq 0$ for a.e. $x \in \Omega$ and $|t| \leq \delta$; or

$\left(T_{3}\right) \frac{|t|^{r}}{r}+H(x, t) \geq c|t|^{s}$ for a.e. $x \in \Omega$ and all $t \in \mathbb{R}$ for some $s \in(p, q)$ and $c>0$.

Recently, Liu and Dai [1] investigated the sign-changing ground state solution of $(P)$ under $\left(h_{1}\right),\left(h_{2}\right),\left(h_{3}\right)$, and

$\left(h_{4}\right)^{\prime}$ the function $t \mapsto \frac{f(x, t)}{|t|^{q-1}}$ is strictly increasing on $(-\infty, 0) \cup(0,+\infty)$.

Additionally, Liu and Dai [9] also obtained the existence of at least three ground state solutions of $(P)$ by using the strong maximum principle for the homogeneous doublephase problem.

It is a well-known consequence of $\left(h_{4}\right)^{\prime}$ that there is unique $t_{u}>0$ such that $t_{u} u \in \mathbb{N}_{0}$ for every $u \in W_{0}^{1, H}(\Omega) \backslash\{0\}$, which implies that $\varphi$ has at most one minimizer on $\mathbb{M}_{0}$. Moreover, $\left(h_{4}\right)^{\prime}$ plays a crucial role in [1]. In fact, condition $\left(h_{4}\right)^{\prime}$ implies that every minimizer of $\varphi$ on $\mathbb{M}_{0}$ is a critical point. However, if $t \mapsto \frac{f(x, t)}{|t|^{q-1}}$ is nonstrictly increasing, then $t_{u}$ and minimizer of $\varphi$ on $\mathbb{M}_{0}$ may not be unique, and their arguments become invalid.

Motivated by the aforementioned works, in the present paper, our goal is to generalize the results mentioned to $(P)$ under a weaker assumption. Precisely, we obtain following results.

Theorem 1.1 Assume that $\left(h_{1}\right)-\left(h_{4}\right)$ hold. Then problem $(P)$ has a sign-changing solution $u_{0} \in \mathbb{M}_{0}$ such that

$$
\varphi\left(u_{0}\right)=\inf _{u \in \mathbb{M}_{0}} \varphi(u)
$$

Furthermore, suppose that

$$
\frac{1}{q} f(x, t) t-F(x, t)>0, \quad \forall x \in \Omega, t \neq 0,
$$

then $u_{0}$ has precisely two nodal domains.

Theorem 1.2 Assume that $\left(h_{1}\right)-\left(h_{4}\right)$ hold. Then $m_{0} \geq 2 n_{0}$.

The rest of this paper is organized as follows. In Sect. 2, we present some necessary preliminary knowledge on space $W_{0}^{1, H}(\Omega)$. In Sect. 3, we give some preliminary lemmas needed for the proofs of our main results. We complete the proofs of Theorems 1.1-1.2 in Sect. 4. 


\section{Preliminaries}

To discuss problem $(P)$, we need some facts on the space $W_{0}^{1, H}(\Omega)$, which is called the Musielak-Orlicz-Sobolev space. For this reason, we recall some properties involving the Musielak-Orlicz spaces, which can be found in [10-14] and references therein.

Denote by $N(\Omega)$ the set of all generalized $N$-functions. For $1<p<q$ and $0 \leq a(\cdot) \in$ $L^{1}(\Omega)$, we define

$$
H(x, t)=t^{p}+a(x) t^{q}, \quad(x, t) \in \Omega \times[0,+\infty) .
$$

It is clear that $H \in N(\Omega)$ is locally integrable and

$$
H(x, 2 t) \leq 2^{q} H(x, t), \quad(x, t) \in \Omega \times[0,+\infty),
$$

which is called condition $\left(\triangle_{2}\right)$.

The Musielak-Orlicz space $L^{H}(\Omega)$ is defined by

$$
L^{H}(\Omega)=\left\{u: \Omega \rightarrow \mathbb{R} \text { measurable }: \int_{\Omega} H(x,|u|) d x<+\infty\right\},
$$

endowed with the Luxemburg norm

$$
|u|_{H}=\inf \left\{\lambda>0: \int_{\Omega} H\left(x,\left|\frac{u}{\lambda}\right|\right) d x \leq 1\right\}
$$

The Musielak-Orlicz-Sobolev space $W^{1, H}(\Omega)$ is defined by

$$
W^{1, H}(\Omega)=\left\{u \in L^{H}(\Omega):|\nabla u| \in L^{H}(\Omega)\right\}
$$

and is equipped with the norm

$$
\|u\|=|u|_{H}+|\nabla u|_{H} .
$$

We denote by $W_{0}^{1, H}(\Omega)$ the completion of $C_{0}^{\infty}(\Omega)$ in $W^{1, H}(\Omega)$. With these norms, the spaces $L^{H}(\Omega), W_{0}^{1, H}(\Omega)$ and $W^{1, H}(\Omega)$ are separable reflexive Banach spaces; see [10] for the details.

Proposition 2.1 ([1, Proposition 2.1]) Set $\rho_{H}(u)=\int_{\Omega}\left(|u|^{p}+a(x)|u|^{q}\right) d x$. For $u \in L^{H}(\Omega)$, we have:

(i) For $u \neq 0,|u|_{H}=\lambda \Leftrightarrow \rho_{H}\left(\frac{u}{\lambda}\right)=1$;

(ii) $|u|_{H}<1(=1$; $>1) \Leftrightarrow \rho_{H}(u)<1(=1$; $>1)$;

(iii) If $|u|_{H} \geq 1$, then $|u|_{H}^{p} \leq \rho_{H}(u) \leq|u|_{H}^{q}$;

(iv) If $|u|_{H} \leq 1$, then $|u|_{H}^{q} \leq \rho_{H}(u) \leq|u|_{H}^{p}$.

Proposition 2.2 ([11, Propositions 2.15 and 2.18])

(1) If $1 \leq \vartheta \leq p^{*}$, then the embedding from $W_{0}^{1, H}(\Omega)$ to $L^{\vartheta}(\Omega)$ is continuous. In particular, if $\vartheta \in\left[1, p^{*}\right)$, then the embedding $W_{0}^{1, H}(\Omega) \hookrightarrow L^{\vartheta}(\Omega)$ is compact. 
(2) Assume that (2) holds. Then the Poincarés inequality holds, that is, there exists a positive constant $C_{0}$ such that

$$
|u|_{H} \leq C_{0}|\nabla u|_{H}, \quad u \in W_{0}^{1, H}(\Omega)
$$

By this lemma there exists $c_{\vartheta}>0$ such that

$$
|u|_{\vartheta} \leq c_{\vartheta}\|u\|, \quad \forall u \in W_{0}^{H}(\Omega)
$$

where $|u|_{s}$ denotes the usual norm in $L^{\vartheta}(\Omega)$ for $1 \leq \vartheta<p^{*}$. It follows from (2) of Proposition 2.2 that $|\nabla u|_{H}$ is an equivalent norm in $W_{0}^{1, H}(\Omega)$. We will use the equivalent norm in the following discussion and write $\|u\|=|\nabla u|_{H}$ for simplicity.

To discuss problem $(P)$, we need to define a functional in $W_{0}^{1, H}(\Omega)$ :

$$
J(u)=\int_{\Omega}\left(\frac{1}{p}|\nabla u|^{p}+\frac{a(x)}{q}|\nabla u|^{q}\right) d x .
$$

We know that (see $\left[15\right.$, p. 63, example]) $J \in C^{1}\left(W_{0}^{1, H}(\Omega), \mathbb{R}\right)$ and the double-phase operator $-\operatorname{div}\left(|\nabla u|^{p-2} \nabla u+a(x)|\nabla u|^{q-2} \nabla u\right)$ is the derivative operator of $J$ in the weak sense. We denote $L=J^{\prime}: W_{0}^{1, H}(\Omega) \rightarrow\left(W_{0}^{1, H}(\Omega)\right)^{*}$. Then

$$
\langle L(u), v\rangle=\int_{\Omega}\left(|\nabla u|^{p-2} \nabla u \cdot \nabla v+a(x)|\nabla u|^{q-2} \nabla u \cdot \nabla v\right) d x
$$

for all $u, v \in W_{0}^{1, H}(\Omega)$. Here $\left(W_{0}^{1, H}(\Omega)\right)^{*}$ denotes the dual space of $W_{0}^{1, H}(\Omega)$, and $\langle\cdot, \cdot\rangle$ denotes the pairing between $W_{0}^{1, H}(\Omega)$ and $\left(W_{0}^{1, H}(\Omega)\right)^{*}$. Then we have the following:

Proposition 2.3 ([1, Proposition 3.1]) Let $E=W_{0}^{1, H}(\Omega)$, and let $L$ be as before. Then

(1) $L: E \rightarrow E^{*}$ a continuous, bounded, and strictly monotone operator.

(2) $L: E \rightarrow E^{*}$ is a mapping of type $(S)_{+}$, that is, if $u_{n} \rightarrow u$ in $E$ and $\lim \sup _{n \rightarrow+\infty}\left\langle L\left(u_{n}\right)-L(u), u_{n}-u\right\rangle \leq 0$, then $u_{n} \rightarrow u$ in $E$.

(3) $L: E \rightarrow E^{*}$ is a homeomorphism.

\section{Some preliminary lemmas}

In this section, we give some preliminary lemmas crucial for proving our results.

Lemma 3.1 If assumptions $\left(h_{1}\right)-\left(h_{4}\right)$ hold, then

$$
\begin{aligned}
& \varphi(u) \geq \varphi\left(s u^{+}+t u^{-}\right)+\frac{1-s^{q}}{q}\left\langle\varphi^{\prime}(u), u^{+}\right\rangle+\frac{1-t^{q}}{q}\left\langle\varphi^{\prime}(u), u^{-}\right\rangle \\
&+\int_{\Omega} g(s)\left|\nabla u^{+}\right|^{p} d x+\int_{\Omega} g(t)\left|\nabla u^{-}\right|^{p} d x, \\
& \forall u=u^{+}+u^{-} \in E, s, t \geq 0
\end{aligned}
$$

where $g(\tau)=\frac{1-\tau^{p}}{p}-\frac{1-\tau^{q}}{q}, \tau \geq 0$. 
Proof By condition $\left(h_{4}\right)$ we have

$$
\begin{aligned}
& \frac{1-t^{q}}{q} \tau f(x, \tau)+F(x, t \tau)-F(x, \tau) \\
& =\int_{t}^{1} f(x, \tau) s^{q-1} \tau d s-\int_{t}^{1} f(x, \tau s) \tau d s \\
& =\int_{t}^{1}\left[\frac{f(x, \tau)}{|\tau|^{q-1}}-\frac{f(x, \tau s)}{|\tau s|^{q-1}}\right] s^{q-1}|\tau|^{q-1} \tau d s \\
& \geq 0, \quad t \geq 0, \tau \in \mathbb{R} \backslash\{0\} .
\end{aligned}
$$

Clearly, $g(t) \geq g(1)=0$ for any $t \geq 0$. Hence from (6) it follows that

$$
\begin{aligned}
\varphi(u) & -\varphi\left(s u^{+}+t u^{-}\right) \\
= & \int_{\Omega}\left(\frac{1}{p}\left|\nabla u^{+}\right|^{p}+\frac{a(x)}{q}\left|\nabla u^{+}\right|^{q}\right) d x-\int_{\Omega} F\left(x, u^{+}\right) d x \\
& +\int_{\Omega}\left(\frac{1}{p}\left|\nabla u^{-}\right|^{p}+\frac{a(x)}{q}\left|\nabla u^{-}\right|^{q}\right) d x-\int_{\Omega} F\left(x, u^{-}\right) d x \\
& -\int_{\Omega}\left(\frac{s^{p}}{p}\left|\nabla u^{+}\right|^{p}+\frac{a(x) s^{q}}{q}\left|\nabla u^{+}\right|^{q}\right) d x+\int_{\Omega} F\left(x, s u^{+}\right) d x \\
& -\int_{\Omega}\left(\frac{t^{p}}{p}\left|\nabla u^{-}\right|^{p}+\frac{a(x) t^{q}}{q}\left|\nabla u^{-}\right|^{q}\right) d x+\int_{\Omega} F\left(x, t u^{-}\right) d x \\
& -\frac{1-s^{q}}{q}\left\langle\varphi^{\prime}(u), u^{+}\right\rangle-\frac{1-t^{q}}{q}\left\langle\varphi^{\prime}(u), u^{-}\right\rangle \\
& +\frac{1-s^{q}}{q}\left\langle\varphi^{\prime}(u), u^{+}\right\rangle+\frac{1-t^{q}}{q}\left\langle\varphi^{\prime}(u), u^{-}\right\rangle \\
= & \frac{1-s^{q}}{q}\left\langle\varphi^{\prime}(u), u^{+}\right\rangle+\frac{1-t^{q}}{q}\left\langle\varphi^{\prime}(u), u^{-}\right\rangle \\
& +\int_{\Omega} g(s)\left|\nabla u^{+}\right|^{p} d x+\int_{\Omega} g(t)\left|\nabla u^{-}\right|^{p} d x \\
& +\frac{1-s^{q}}{q}\left\langle\varphi^{\prime}(u), u^{+}\right\rangle+\frac{1-t^{q}}{q}\left\langle\varphi^{\prime}(u), u^{-}\right\rangle \\
& +\int_{\Omega}\left[\frac{1-s^{q}}{q} f\left(x, u^{+}\right) u^{+}+F\left(x, s u^{+}\right)-F\left(x, u^{+}\right)\right] d x \\
& \left.+\frac{1-t^{q}}{q} f\left(x, u^{-}\right) u^{-}+F\left(x, t u^{-}\right)-F\left(x, u^{-}\right)\right] d x
\end{aligned}
$$

The proof is completed.

From Lemma 3.1 we immediately have the following two corollaries.

Corollary 3.2 Assume that $\left(h_{1}\right)-\left(h_{4}\right)$ hold. If $u=u^{+}+u^{-} \in \mathbb{M}_{0}$, then

$$
\varphi(u)=\varphi\left(u^{+}+u^{-}\right)=\max _{s, t \geq 0} \varphi\left(s u^{+}+t u^{-}\right) .
$$


Corollary 3.3 Assume that $\left(h_{1}\right)-\left(h_{4}\right)$ hold. If $u \in \mathbb{N}_{0}$, then

$$
\varphi(u)=\max _{t \geq 0} \varphi(t u)
$$

Lemma 3.4 Assume that $\left(h_{1}\right)-\left(h_{3}\right)$ and $\left(h_{4}\right)^{\prime}$ hold. If $u \in E$ and $u^{ \pm} \neq 0$, then there exists a unique pair $\left(s_{u}, t_{u}\right)$ of positive numbers such that

$$
s_{u} u^{+}+t_{u} u^{-} \in \mathbb{M}_{0}
$$

Proof For any $u \in E$ with $u^{ \pm} \neq 0$, we consider the functions $g(s, t), h(s, t):[0,+\infty) \times$ $[0,+\infty) \rightarrow \mathbb{R}$ given by

$$
g(s, t)=\left\langle\varphi^{\prime}\left(s u^{+}+t u^{-}\right), s u^{+}\right\rangle \quad \text { and } \quad h(s, t)=\left\langle\varphi^{\prime}\left(s u^{+}+t u^{-}\right), t u^{-}\right\rangle
$$

We directly compute that

$$
\begin{aligned}
g(s, t) & =\left\langle\varphi^{\prime}\left(s u^{+}+t u^{-}\right), s u^{+}\right\rangle \\
& =\int_{\Omega}\left(s^{p}\left|\nabla u^{+}\right|^{p}+a(x) s^{q}\left|\nabla u^{+}\right|^{q}\right) d x-\int_{\Omega} f\left(x, s u^{+}\right) s u^{+} d x, \\
h(s, t) & =\left\langle\varphi^{\prime}\left(s u^{+}+t u^{-}\right), t u^{-}\right\rangle \\
& =\int_{\Omega}\left(t^{p}\left|\nabla u^{-}\right|^{p}+a(x) t^{q}\left|\nabla u^{-}\right|^{q}\right) d x-\int_{\Omega} f\left(x, t u^{-}\right) t u^{-} d x .
\end{aligned}
$$

Using assumptions $\left(h_{1}\right)$ and $\left(h_{2}\right)$, we deduce that, for any $\varepsilon>0$, there is $C_{\varepsilon}>0$ such that, for all $(x, t) \in \Omega \times \mathbb{R}$,

$$
\begin{aligned}
& |f(x, t)| \leq \varepsilon|t|^{p-1}+C_{\varepsilon}|t|^{r-1}, \\
& |F(x, t)| \leq \varepsilon|t|^{p}+C_{\varepsilon}|t|^{r},
\end{aligned}
$$

where $r \in\left[1, p^{*}\right)$ was given in $\left(h_{2}\right)$.

Thus, for $s>0$ sufficiently small, by (8) and Proposition 2.2(2) we have

$$
\begin{aligned}
g(s, t)= & \int_{\Omega}\left(s^{p}\left|\nabla u^{+}\right|^{p}+a(x) s^{q}\left|\nabla u^{+}\right|^{q}\right) d x-\int_{\Omega} f\left(x, s u^{+}\right) s u^{+} d x \\
\geq & s^{q} \int_{\Omega}\left(\left|\nabla u^{+}\right|^{p}+a(x)\left|\nabla u^{+}\right|^{q}\right) d x \\
& -\int_{\Omega}\left(\varepsilon s^{p}\left|u^{+}\right|^{p}+C_{\varepsilon} s^{r}\left|u^{+}\right|^{r}\right) d x \\
\geq & \begin{cases}s^{q}\left\|u^{+}\right\|^{q}-\varepsilon c_{p}^{p} s^{p}\left\|u^{+}\right\|^{p}-C_{\varepsilon} c_{r}^{r} s^{r}\left\|u^{+}\right\|^{r} & \text { if }\left\|u^{+}\right\|<1, \\
s^{q}\left\|u^{+}\right\|^{p}-\varepsilon c_{p}^{p} s^{p}\left\|u^{+}\right\|^{p}-C_{\varepsilon} c_{r}^{r} s^{r}\left\|u^{+}\right\|^{r} & \text { if }\left\|u^{+}\right\|>1,\end{cases}
\end{aligned}
$$

and

$$
\begin{aligned}
h(s, t) & =\int_{\Omega}\left(t^{p}\left|\nabla u^{-}\right|^{p}+a(x) t^{q}\left|\nabla u^{-}\right|^{q}\right) d x-\int_{\Omega} f\left(x, t u^{-}\right) t u^{-} d x \\
& \geq t^{q} \int_{\Omega}\left(\left|\nabla u^{-}\right|^{p}+a(x)\left|\nabla u^{-}\right|^{q}\right) d x
\end{aligned}
$$




$$
\begin{aligned}
& -\int_{\Omega}\left(\varepsilon t^{p}\left|u^{-}\right|^{p}+C_{\varepsilon} t^{r}\left|u^{-}\right|^{r}\right) d x \\
\geq & \begin{cases}t^{q}\left\|u^{-}\right\|^{q}-\varepsilon c_{p}^{p} t^{p}\left\|u^{-}\right\|^{p}-C_{\varepsilon} c_{r}^{r} t^{r}\left\|u^{-}\right\|^{r} & \text { if }\left\|u^{-}\right\|<1, \\
t^{q}\left\|u^{-}\right\|^{p}-\varepsilon c_{p}^{p} t^{p}\left\|u^{-}\right\|^{p}-C_{\varepsilon} c_{r}^{r} t^{r}\left\|u^{-}\right\|^{r} & \text { if }\left\|u^{-}\right\|>1 .\end{cases}
\end{aligned}
$$

By (9), (10), and the arbitrariness of $\varepsilon$, it is easy to prove that $g(s, s)>0$ and $h(s, s)>0$ for $s>0$ small.

Moreover, using (6), we have

$$
\frac{1}{q} \tau f(x, \tau)-F(x, \tau) \geq 0, \quad \tau \in \mathbb{R} \backslash\{0\} .
$$

Hence by $(11)$ and $\left(h_{3}\right)$ we have that, for $s>1$,

$$
\begin{aligned}
g(s, t) & =\int_{\Omega}\left(s^{p}\left|\nabla u^{+}\right|^{p}+a(x) s^{q}\left|\nabla u^{+}\right|^{q}\right) d x-\int_{\Omega} f\left(x, s u^{+}\right) s u^{+} d x \\
& \leq s^{q} \int_{\Omega}\left(\left|\nabla u^{+}\right|^{p}+a(x)\left|\nabla u^{+}\right|^{q}\right) d x-q \int_{\Omega} F\left(x, s u^{+}\right) d x \\
& =s^{q} \int_{\Omega}\left(\left|\nabla u^{+}\right|^{p}+a(x)\left|\nabla u^{+}\right|^{q}\right) d x-q \int_{\Omega} \frac{F\left(x, s u^{+}\right)}{\left|s u^{+}\right|^{q}}\left|s u^{+}\right|^{q} d x \\
& =s^{q}\left(\int_{\Omega}\left(\left|\nabla u^{+}\right|^{p}+a(x)\left|\nabla u^{+}\right|^{q}\right) d x-q \int_{u^{+} \neq 0} \frac{F\left(x, s u^{+}\right)}{\left|s u^{+}\right|^{q}}\left|u^{+}\right|^{q} d x\right)
\end{aligned}
$$

and, for $t>1$,

$$
\begin{aligned}
g(s, t) & =\int_{\Omega}\left(t^{p}\left|\nabla u^{-}\right|^{p}+a(x) t^{q}\left|\nabla u^{-}\right|^{q}\right) d x-\int_{\Omega} f\left(x, t u^{-}\right) t u^{-} d x \\
& \leq t^{q} \int_{\Omega}\left(\left|\nabla u^{-}\right|^{p}+a(x)\left|\nabla u^{-}\right|^{q}\right) d x-q \int_{\Omega} F\left(x, t u^{+}\right) d x \\
& =t^{q} \int_{\Omega}\left(\left|\nabla u^{-}\right|^{p}+a(x)\left|\nabla u^{-}\right|^{q}\right) d x-q \int_{\Omega} \frac{F\left(x, t u^{-}\right)}{\left|t u^{-}\right|^{q}}\left|t u^{-}\right|^{q} d x \\
& =t^{q}\left(\int_{\Omega}\left(\left|\nabla u^{-}\right|^{p}+a(x)\left|\nabla u^{-}\right|^{q}\right) d x-q \int_{u^{-} \neq 0} \frac{F\left(x, t u^{+}\right)}{\left|t u^{-}\right|^{q}}\left|u^{-}\right|^{q} d x\right),
\end{aligned}
$$

which yields that $g(t, t)<0$ and $h(t, t)<0$ for $t>0$ large. Thus there are $0<T<R$ such that

$$
g(T, T), h(T, T)>0 \quad \text { and } \quad g(R, R), h(R, R)<0 .
$$

This fact, combined with (7), implies that

$$
g(T, t)=g(T, T)>0, \quad g(R, t)=g(R, R)<0, \quad t \in[r, R],
$$

and

$$
h(T, t)=h(T, T)>0, \quad h(R, t)=h(R, R)<0, \quad t \in[r, R] .
$$

So, by the Miranda theorem in [16] we can find $\left(s_{u}, t_{u}\right) \in(T, R) \times(T, R)$ such that $g\left(s_{u}, t_{u}\right)=$ $h\left(s_{u}, t_{u}\right)=0$. Therefore $s_{u} u^{+}+t_{u} u^{-} \in \mathbb{M}_{0}$. 
Next, we prove the uniqueness. Let $\left(s_{i}, t_{i}\right)$ be such that $s_{i} u^{+}+t_{i} u^{-} \in \mathbb{M}_{0}, i=1,2$, that is,

$$
g\left(s_{1}, t_{1}\right)=h\left(s_{1}, t_{1}\right)=g\left(s_{2}, t_{2}\right)=h\left(s_{2}, t_{2}\right)=0 .
$$

Then from (5), (7), and (15) it follows that

$$
\begin{aligned}
\varphi\left(s_{1} u^{+}+t_{1} u^{-}\right) \geq & \frac{s_{1}^{q}-s_{2}^{q}}{q s_{1}^{q}}\left\langle\varphi^{\prime}\left(s_{1} u^{+}+t_{1} u^{-}\right), s_{1} u^{+}\right\rangle \\
& +\frac{t_{1}^{q}-t_{2}^{q}}{q t_{1}^{q}}\left\langle\varphi^{\prime}\left(s_{1} u^{+}+t_{1} u^{-}\right), t_{1} u^{-}\right\rangle \\
& +\varphi\left(s_{2} u^{+}+t_{2} u^{-}\right) \\
& +\left(\frac{s_{1}^{p}-s_{2}^{p}}{p}-\frac{s_{1}^{q}-s_{2}^{q}}{q s_{1}^{q}} s_{1}^{p}\right) \int_{\Omega}\left|\nabla u^{+}\right|^{p} d x \\
& +\left(\frac{t_{1}^{p}-t_{2}^{p}}{p}-\frac{t_{1}^{q}-t_{2}^{q}}{q t_{1}^{q}} t_{1}^{p}\right) \int_{\Omega}\left|\nabla u^{-}\right|^{p} d x \\
= & \varphi\left(s_{2} u^{+}+t_{2} u^{-}\right) \\
& +\left(\frac{s_{1}^{p}-s_{2}^{p}}{p}-\frac{s_{1}^{q}-s_{2}^{q}}{q s_{1}^{q}} s_{1}^{p}\right) \int_{\Omega}\left|\nabla u^{+}\right|^{p} d x \\
& +\left(\frac{t_{1}^{p}-t_{2}^{p}}{p}-\frac{t_{1}^{q}-t_{2}^{q}}{q t_{1}^{q}} t_{1}^{p}\right) \int_{\Omega}\left|\nabla u^{-}\right|^{p} d x
\end{aligned}
$$

and

$$
\begin{aligned}
\varphi\left(s_{2} u^{+}+t_{2} u^{-}\right) \geq & \frac{s_{2}^{q}-s_{1}^{q}}{q s_{2}^{q}}\left\langle\varphi^{\prime}\left(s_{2} u^{+}+t_{2} u^{-}\right), s_{2} u^{+}\right\rangle \\
& +\frac{t_{2}^{q}-t_{1}^{q}}{q t_{2}^{q}}\left\langle\varphi^{\prime}\left(s_{2} u^{+}+t_{2} u^{-}\right), t_{2} u^{-}\right\rangle \\
& +\varphi\left(s_{1} u^{+}+t_{1} u^{-}\right) \\
& +\left(\frac{s_{2}^{p}-s_{1}^{p}}{p}-\frac{s_{2}^{q}-s_{1}^{q}}{q s_{2}^{q}} s_{2}^{p}\right) \int_{\Omega}\left|\nabla u^{+}\right|^{p} d x \\
& +\left(\frac{t_{2}^{p}-t_{1}^{p}}{p}-\frac{t_{2}^{q}-t_{1}^{q}}{q t_{2}^{q}} t_{2}^{p}\right) \int_{\Omega}\left|\nabla u^{-}\right|^{p} d x \\
= & \varphi\left(s_{1} u^{+}+t_{1} u^{-}\right) \\
& +\left(\frac{s_{2}^{p}-s_{1}^{p}}{p}-\frac{s_{2}^{q}-s_{1}^{q}}{q s_{2}^{q}} s_{2}^{p}\right) \int_{\Omega}\left|\nabla u^{+}\right|^{p} d x \\
& +\left(\frac{t_{2}^{p}-t_{1}^{p}}{p}-\frac{t_{2}^{q}-t_{1}^{q}}{q t_{2}^{q}} t_{2}^{p}\right) \int_{\Omega}\left|\nabla u^{-}\right|^{p} d x .
\end{aligned}
$$

Both (16) and (17) imply that $s_{1}=s_{2}$ and $t_{1}=t_{2}$, which in turn implies that $\left(s_{u}, t_{u}\right)$ is the unique pair of positive numbers such that $s_{u} u^{+}+t_{u} u^{-} \in \mathbb{M}_{0}$. We end the proof.

Furthermore we have the following: 
Lemma 3.5 Assume that $\left(h_{1}\right)-\left(h_{3}\right)$ and $\left(h_{4}\right)^{\prime}$ hold. Then

$$
m_{0}=\inf _{u \in \mathbb{M}_{0}} \varphi(u)=\inf _{u \in E, u^{ \pm} \neq 0} \max _{s, t \geq 0} \varphi\left(s u^{+}+t u^{-}\right) .
$$

Proof By Corollary 3.2 we conclude that

$$
\begin{aligned}
\inf _{u \in E, u^{ \pm} \neq 0} \max _{s, t \geq 0} \varphi\left(s u^{+}+t u^{-}\right) & \leq \inf _{u \in \mathbb{M}_{0}} \max _{s, t \geq 0} \varphi\left(s u^{+}+t u^{-}\right) \\
& =\inf _{u \in \mathbb{M}_{0}} \varphi(u)=m_{0} .
\end{aligned}
$$

Moreover, for any $u \in E$ with $u^{ \pm} \neq 0$, from Lemma 3.4 we deduce that

$$
\max _{s, t \geq 0} \varphi\left(s u^{+}+t u^{-}\right) \geq \varphi\left(s_{u} u^{+}+t_{u} u^{-}\right) \geq \inf _{u \in \mathbb{M}_{0}} \varphi(u)=m_{0},
$$

which implies

$$
\inf _{u \in E, u^{ \pm} \neq 0} \max _{s, t \geq 0} \varphi\left(s u^{+}+t u^{-}\right) \geq \inf _{u \in \mathbb{M}_{0}} \varphi(u)=m_{0} .
$$

Therefore the conclusion directly follows from (18) and (19).

Lemma 3.6 Assume that $\left(h_{1}\right)-\left(h_{3}\right)$ and $\left(h_{4}\right)^{\prime}$ hold. Then $m_{0}>0$ can be achieved.

Proof Firstly, we will show that $m_{0}>0$. Indeed, for every $u \in \mathbb{M}_{0}$, we have $u \in \mathbb{N}_{0}$ and $\left\langle\varphi^{\prime}(u), u\right\rangle=0$. Then by $\left(h_{1}\right)-\left(h_{2}\right)$ and Propositions 2.1 and 2.2 we get

$$
\begin{aligned}
& \varepsilon c_{p}^{p}\|u\|^{p}+C_{\varepsilon} c_{r}^{r}\|u\|^{r} \\
& \quad \geq \varepsilon|u|_{p}^{p}+C_{\varepsilon}|u|_{r}^{r} \\
& \quad \geq \int_{\Omega} f(x, u) u d x \\
& \quad=\int_{\Omega}\left(|\nabla u|^{p}+a(x)|\nabla u|^{q}\right) d x \\
& \quad \geq \begin{cases}\|u\|^{q} & \text { if }\|u\|<1, \\
\|u\|^{p} & \text { if }\|u\|>1 .\end{cases}
\end{aligned}
$$

Thus, for any $u \in \mathbb{N}_{0}$ with $\|u\|<1$, we have that

$$
\frac{1}{2}\|u\|^{q} \leq C_{\varepsilon} c_{r}^{r}\|u\|^{r},
$$

which implies that

$$
\|u\| \geq\left(\frac{1}{2 C_{\varepsilon} c_{r}^{r}}\right)^{\frac{1}{r-q}}=: \alpha_{0} .
$$

Therefore we obtain that $m_{0}=\inf _{u \in \mathbb{M}_{0}} \varphi(u) \geq \alpha_{0}>0$. 
It remains to prove that $u_{0} \in \mathbb{M}_{0}$ and $\varphi\left(u_{0}\right)=m_{0}$. Let $\left\{u_{n}\right\} \subset \mathbb{M}_{0}$ be a sequence of functions such that $\varphi\left(u_{n}\right) \rightarrow m_{0}$ as $n \rightarrow+\infty$. Firstly, we claim that $\left\{u_{n}\right\}$ is bounded. Suppose, by contradiction, that $\left\|u_{n}\right\| \rightarrow+\infty$ and let $v_{n}=\frac{u_{n}}{\left\|u_{n}\right\|}$. Without loss of generality, we may assume that $v_{n} \rightarrow v$ in $E$. By the Sobolev embedding theorem we have

$$
v_{n} \rightarrow v \quad \text { in } L^{\vartheta}(\Omega), 1 \leq \vartheta<p^{*}, \quad v_{n} \rightarrow v \quad \text { a.e. on } \Omega
$$

If $v=0$, then $v_{n} \rightarrow 0$ in $L^{\vartheta}(\Omega)$ for $1 \leq \vartheta<p^{*}$. Fix $R>\left[q\left(m_{0}+1\right)\right]^{\frac{1}{p}}(>1)$. By $\left(h_{1}\right)-\left(h_{2}\right)$ there exists $C_{1}>0$ such that

$$
F(x, t) \leq|t|^{p}+C_{1}|t|^{r}, \quad x \in \Omega, t \in \mathbb{R}
$$

Then we have that

$$
\limsup _{n \rightarrow \infty} \int_{\Omega} F\left(x, R v_{n}\right) d x \leq R^{p} \lim _{n \rightarrow \infty}\left(\left|v_{n}\right|_{p}^{p}+C_{1} R^{r}\left|v_{n}\right|_{r}^{r}\right)=0 .
$$

Let $t_{n}=\frac{R}{\left\|u_{n}\right\|}$. Hence by (20) and Corollary 3.3 we get that

$$
\begin{aligned}
m_{0}+o(1) & =\varphi\left(u_{n}\right) \\
& \geq \varphi\left(t_{n} u_{n}\right) \\
& =\varphi\left(R v_{n}\right) \\
& =\int_{\Omega}\left(\frac{1}{p} R^{p}\left|\nabla v_{n}\right|^{p}+\frac{a(x)}{q} R^{q}\left|\nabla v_{n}\right|^{q}\right) d x-\int_{\Omega} F\left(x, R v_{n}\right) d x \\
& \geq \frac{1}{q} R^{p}-\int_{\Omega} F\left(x, R v_{n}\right) d x \\
& \geq \frac{1}{q} R^{p}+o(1) \\
& >m_{0}+1+o(1),
\end{aligned}
$$

which yields a contradiction. Thus $v \neq 0$.

For $x \in\left\{y \in \mathbb{R}^{N}: v(y) \neq 0\right\}$, it is clear that $\lim _{n \rightarrow+\infty}\left|u_{n}(x)\right|=+\infty$. By hypotheses $\left(h_{1}\right)$ and $\left(h_{2}\right)$ we can find $C_{2} \in \mathbb{R}$ such that

$$
F(x, t) \geq C_{2}, \quad(x, t) \in \Omega \times \mathbb{R} .
$$

Hence by using (21), $\left(h_{3}\right)$, Proposition 2.1, and Fatou's lemma we have

$$
\begin{aligned}
0 & =\lim _{n \rightarrow+\infty} \frac{m+o(1)}{\left\|u_{n}\right\|^{q}}=\lim _{n \rightarrow+\infty} \frac{\varphi\left(u_{n}\right)}{\left\|u_{n}\right\|^{q}} \\
& \leq \lim _{n \rightarrow+\infty}\left[\frac{1}{p} \frac{\int_{\Omega}\left(\left|\nabla u_{n}\right|^{p}+a(x)\left|\nabla u_{n}\right|^{q}\right) d x}{\left\|u_{n}\right\|^{q}}-\int_{\Omega} \frac{F\left(x, u_{n}\right)}{\left\|u_{n}\right\|^{q}} d x\right] \\
& \leq \frac{1}{p}-\lim _{n \rightarrow+\infty} \int_{\Omega} \frac{F\left(x, u_{n}\right)}{\left\|u_{n}\right\|^{q}} d x \\
& =\frac{1}{p}-\lim _{n \rightarrow+\infty} \int_{\Omega} \frac{F\left(x, u_{n}\right)-C_{2}}{\left\|u_{n}\right\|^{q}} d x
\end{aligned}
$$




$$
\begin{aligned}
& \leq \frac{1}{p}-\liminf _{n \rightarrow+\infty} \int_{\Omega} \frac{F\left(x, u_{n}\right)-C_{2}}{\left\|u_{n}\right\|^{q}} d x \\
& =\frac{1}{p}-\liminf _{n \rightarrow+\infty} \int_{\Omega} \frac{F\left(x, u_{n}\right)}{\left\|u_{n}\right\|^{q}} d x \\
& \leq \frac{1}{p}-\int_{\Omega} \liminf _{n \rightarrow+\infty} \frac{F\left(x, u_{n}(x)\right)}{\left|u_{n}(x)\right|^{q}}\left|v_{n}(x)\right|^{q} d x \\
& =-\infty .
\end{aligned}
$$

This contradiction shows that $\left\{u_{n}\right\}$ is bounded in $E$. Going if necessary to a subsequence, we can assume that $u_{n}^{ \pm} \rightarrow u_{0}^{ \pm}$in $E$. Then $u_{n}^{ \pm} \rightarrow u_{0}^{ \pm}$in $L^{\vartheta}(\Omega)$ for $\vartheta \in\left[1, p^{*}\right)$ and $u_{n} \rightarrow u_{0}$ a.e. on $\Omega$.

Our next goal is to prove that $u_{0} \in \mathbb{M}_{0}$ and $\varphi\left(u_{0}\right)=m_{0}$. Firstly, we claim that $\inf _{u \in \mathbb{N}_{0}} \varphi(u)>$ 0 . Indeed, for every $u \in \mathbb{N}_{0}$, we have $\left\langle\varphi^{\prime}(u), u\right\rangle=0$. Then by $\left(h_{1}\right),\left(h_{2}\right)$, and Propositions 2.1 and 2.2 we get

$$
\begin{aligned}
& \varepsilon c_{p}^{p}\|u\|^{p}+C_{\varepsilon} c_{r}^{r}\|u\|^{r} \\
& \geq \varepsilon|u|_{p}^{p}+C_{\varepsilon}|u|_{r}^{r} \\
& \geq \int_{\Omega} f(x, u) u d x \\
& =\int_{\Omega}\left(|\nabla u|^{p}+a(x)|\nabla u|^{q}\right) d x \\
& \quad \geq \begin{cases}\|u\|^{q} & \text { if }\|u\|<1, \\
\|u\|^{p} & \text { if }\|u\|>1 .\end{cases}
\end{aligned}
$$

Thus, for any $u \in \mathbb{N}_{0}$ with $\|u\|<1$, we have that

$$
\frac{1}{2}\|u\|^{q} \leq C_{\varepsilon} c_{r}^{r}\|u\|^{r}
$$

which implies that $\|u\| \geq \alpha_{0}$. This implies that $\inf _{u \in \mathbb{N}_{0}} \varphi(u)>0$. Note that $\left\{u_{n}\right\}_{n \in N} \subset \mathbb{M}_{0}$. Then it is obvious that $\left\{u_{n}^{ \pm}\right\}_{n \in N} \subset \mathbb{N}_{0}$, that is,

$$
\int_{\Omega}\left(\left|\nabla u_{n}^{ \pm}\right|^{p}+a(x)\left|\nabla u_{n}^{ \pm}\right|^{q}\right) d x=\int_{\Omega} f\left(x, u_{n}^{ \pm}\right) u_{n}^{ \pm} d x \quad \text { and } \quad\left\|u_{n}^{ \pm}\right\| \geq \alpha_{0}
$$

By $\left(h_{1}\right)$ and $\left(h_{2}\right)$, for any $\varepsilon>0$, there exists $C_{\varepsilon}>0$ such that

$$
|f(x, t)| \leq \varepsilon|t|^{p-1}+C_{\varepsilon}|t|^{r-1}
$$

for all $(x, t) \in \Omega \times \mathbb{R}$, where $r \in\left[1, p^{*}\right)$ was given in $\left(h_{2}\right)$. Thus

$$
\begin{aligned}
& \min \left\{\alpha_{0}^{p}, \alpha_{0}^{q}\right\} \\
& \quad \leq \min \left\{\left\|u_{n}^{ \pm}\right\|^{p},\left\|u_{n}^{ \pm}\right\|^{q}\right\} \\
& \quad \leq \int_{\Omega}\left(\left|\nabla u_{n}^{ \pm}\right|^{p}+a(x)\left|\nabla u_{n}^{ \pm}\right|^{q}\right) d x
\end{aligned}
$$




$$
\begin{aligned}
& =\int_{\Omega} f\left(x, u_{n}^{ \pm}\right) u_{n}^{ \pm} d x \\
& \leq \varepsilon \int_{\Omega}\left|u_{n}^{ \pm}\right|^{p} d x+C_{\varepsilon} \int_{\Omega}\left|u_{n}^{ \pm}\right|^{r} d x .
\end{aligned}
$$

Because of the boundedness of $u_{n}$, there is $C_{1}>0$ such that

$$
\min \left\{\alpha_{0}^{p}, \alpha_{0}^{q}\right\} \leq \varepsilon C_{1}+C_{\varepsilon} \int_{\Omega}\left|u_{n}^{ \pm}\right|^{r} d x
$$

Choosing $\varepsilon=\frac{\min \left\{\alpha_{0}^{p}, \alpha_{0}^{q}\right\}}{2 C_{1}}$, we get

$$
\int_{\Omega}\left|u_{n}^{ \pm}\right|^{r} d x \geq \frac{\min \left\{\alpha_{0}^{p}, \alpha_{0}^{q}\right\}}{2 C_{\varepsilon}}
$$

By the compactness of the embedding $E \hookrightarrow L^{r}(\Omega)$ for $p<q<r<p^{*}$ we get

$$
\int_{\Omega}\left|u_{0}^{ \pm}\right|^{r} d x \geq \frac{\min \left\{\alpha_{0}^{p}, \alpha_{0}^{q}\right\}}{2 C_{\varepsilon}},
$$

which yields $u_{0}^{ \pm} \neq 0$. Moreover, note that $u_{n}^{ \pm} \rightarrow u_{0}^{ \pm}$in $L^{\vartheta}(\Omega), \vartheta \in\left[1, p^{*}\right)$. By conditions $\left(h_{1}\right)$ and $\left(h_{2}\right)$, combined with the Hölder inequality and Lebesgue theorem, we have

$$
\begin{aligned}
& \lim _{n \rightarrow+\infty} \int_{\Omega} f\left(x, u_{n}^{ \pm}\right) u_{n}^{ \pm} d x=\int_{\Omega} f\left(x, u_{0}^{ \pm}\right) u_{0}^{ \pm} d x, \\
& \lim _{n \rightarrow+\infty} \int_{\Omega} F\left(x, u_{n}^{ \pm}\right) d x=\int_{\Omega} F\left(x, u_{0}^{ \pm}\right) d x
\end{aligned}
$$

Hence by the weak lower semicontinuity of the norm we conclude that

$$
\begin{aligned}
\left\langle\varphi^{\prime}\left(u_{0}\right), u_{0}^{ \pm}\right\rangle= & \int_{\Omega}\left(\left|\nabla u_{0}^{ \pm}\right|^{p}+a(x)\left|\nabla u_{0}^{ \pm}\right|^{q}\right) d x-\int_{\Omega} f\left(x, u_{0}^{ \pm}\right) u_{0}^{ \pm} d x \\
\leq & \liminf _{n \rightarrow+\infty} \int_{\Omega}\left(\left|\nabla u_{n}^{ \pm}\right|^{p}+a(x)\left|\nabla u_{n}^{ \pm}\right|^{q}\right) d x \\
& \quad-\lim _{n \rightarrow+\infty} \int_{\Omega} f\left(x, u_{n}^{ \pm}\right) u_{n}^{ \pm} d x \\
= & \liminf _{n \rightarrow+\infty}\left(\varphi^{\prime}\left(u_{n}\right), u_{n}^{ \pm}\right\rangle=0,
\end{aligned}
$$

because $u_{n}^{ \pm} \in \mathbb{N}_{0}$. Thus by Lemma 3.4 there exist $s_{0}, t_{0}>0$ such that $s_{0} u_{0}^{+}+t_{0} u_{0}^{-} \in \mathbb{M}_{0}$. Consequently, from (24) and Lemma 3.1 we have

$$
\begin{aligned}
m_{0} & =\lim _{n \rightarrow+\infty}\left[\varphi\left(u_{n}\right)-\frac{1}{q}\left\langle\varphi^{\prime}\left(u_{n}\right), u_{n}\right\rangle\right] \\
& =\lim _{n \rightarrow+\infty} \int_{\Omega}\left(\frac{1}{p}-\frac{1}{q}\right)\left|\nabla u_{n}\right|^{p} d x+\lim _{n \rightarrow+\infty} \int_{\Omega}\left[\frac{1}{q} f\left(x, u_{n}\right) u_{n}-F\left(x, u_{n}\right)\right] d x \\
& \geq \liminf _{n \rightarrow+\infty} \int_{\Omega}\left(\frac{1}{p}-\frac{1}{q}\right)\left|\nabla u_{n}\right|^{p} d x+\lim _{n \rightarrow+\infty} \int_{\Omega}\left[\frac{1}{q} f\left(x, u_{n}\right) u_{n}-F\left(x, u_{n}\right)\right] d x \\
& \geq \int_{\Omega}\left(\frac{1}{p}-\frac{1}{q}\right)\left|\nabla u_{0}\right|^{p} d x+\int_{\Omega}\left[\frac{1}{q} f\left(x, u_{0}\right) u_{0}-F\left(x, u_{0}\right)\right] d x
\end{aligned}
$$




$$
\begin{aligned}
= & \varphi\left(u_{0}\right)-\frac{1}{q}\left\langle\varphi^{\prime}\left(u_{0}\right), u_{0}\right\rangle \\
\geq & \varphi\left(s_{0} u_{0}^{+}+t_{0} u_{0}^{-}\right)+\frac{1-s_{0}^{q}}{q}\left\langle\varphi^{\prime}\left(u_{0}\right), u_{0}^{+}\right\rangle+\frac{1-t_{0}^{q}}{q}\left\langle\varphi^{\prime}\left(u_{0}\right), u_{0}^{-}\right\rangle \\
& -\frac{1}{q}\left\langle\varphi^{\prime}\left(u_{0}\right), u_{0}\right\rangle \\
= & \varphi\left(s_{0} u_{0}^{+}+t_{0} u_{0}^{-}\right)-\frac{s_{0}^{q}}{q}\left\langle\varphi^{\prime}\left(u_{0}\right), u_{0}^{+}\right\rangle-\frac{t_{0}^{q}}{q}\left\langle\varphi^{\prime}\left(u_{0}\right), u_{0}^{-}\right\rangle \\
\geq & m_{0}-\frac{s_{0}^{q}}{q}\left\langle\varphi^{\prime}\left(u_{0}\right), u_{0}^{+}\right\rangle-\frac{t_{0}^{q}}{q}\left\langle\varphi^{\prime}\left(u_{0}\right), u_{0}^{-}\right\rangle .
\end{aligned}
$$

This shows that

$$
\frac{s_{0}^{q}}{q}\left\langle\varphi^{\prime}\left(u_{0}\right), u_{0}^{+}\right\rangle+\frac{t_{0}^{q}}{q}\left\langle\varphi^{\prime}\left(u_{0}\right), u_{0}^{-}\right\rangle \geq 0
$$

From this and from (25) we conclude that

$$
\left\langle\varphi^{\prime}\left(u_{0}\right), u_{0}^{ \pm}\right\rangle=0 \quad \text { and } \quad \varphi\left(u_{0}\right)=m_{0}
$$

Similarly to the proof of [1, Theorem 1.4], we can prove the following lemma.

Lemma 3.7 Assume that $\left(h_{1}\right)-\left(h_{3}\right)$ and $\left(h_{4}\right)^{\prime}$ hold. If $u_{0} \in \mathbb{M}_{0}$ and $\varphi\left(u_{0}\right)=m_{0}$, then $u_{0}$ is a critical point of $\varphi$.

Proof It is clear that $\left\langle\varphi^{\prime}\left(u_{0}^{ \pm}\right), u_{0}^{ \pm}\right\rangle=0=\left\langle\varphi^{\prime}\left(u_{0}\right), u_{0}^{ \pm}\right\rangle$. It follows from assumption $\left(h_{4}\right)^{\prime}$ that, for $0<s \neq 1$ and $0<t \neq 1$,

$$
\begin{aligned}
\varphi\left(s u_{0}^{+}+t u_{0}^{-}\right) & =\varphi\left(s u_{0}^{+}\right)+\varphi\left(t u_{0}^{-}\right) \\
& <\varphi\left(u_{0}^{+}\right)+\varphi\left(u_{0}^{-}\right) \\
& =\varphi\left(u_{0}\right)=m_{0} .
\end{aligned}
$$

If $\varphi^{\prime}\left(u_{0}\right) \neq 0$, then there exist $\delta>0$ and $v>0$ such that

$$
\left\|v-u_{0}\right\| \leq 3 \delta \quad \Rightarrow \quad\left\|\varphi^{\prime}(v)\right\| \geq v .
$$

Let $D=\left(\frac{1}{2}, \frac{3}{2}\right) \times\left(\frac{1}{2}, \frac{3}{2}\right)$ and $g(s, t)=s u_{0}^{+}+t u_{0}^{-}$. By (26) we have

$$
\beta=\max _{(s, t) \in \partial D} \varphi(g(s, t))<m_{0}
$$

Let $\varepsilon:=\min \left\{\frac{m_{0}-\beta}{4}, \frac{\lambda \delta}{8}\right\}$ and $B(u, \delta):=\{v \in E:\|v-u\| \leq \delta\}$. Then [17, Lemma 2.3] yields a deformation $\eta$ such that

(a) $\eta(1, v)=v$ if $\varphi(v)<m_{0}-2 \varepsilon$ or $\varphi(v)>m_{0}+2 \varepsilon$,

(b) $\eta\left(1, \varphi^{m_{0}+\varepsilon} \cap B(u, \delta)\right) \subset \varphi^{m_{0}-\varepsilon}$, and

(c) $\varphi(\eta(1, v)) \leq \varphi(v)$ for all $v \in E$,

where $\varphi^{m_{0} \pm \varepsilon}:=\left\{v \in E: \varphi(v) \leq m_{0} \pm \varepsilon\right\}$. 
It is easy to see that

$$
\max _{(s, t) \in D} \varphi(\eta(1, g(s, t)))<m_{0}
$$

Next, we show that $\eta(1, g(D)) \cap \mathbb{M}_{0} \neq \emptyset$, contradicting the definition of $m_{0}$. Let $h(s, t)=$ $\eta(1, g(s, t)), \varphi_{0}(s, t)=\left\langle\varphi^{\prime}\left(s u_{0}^{+}\right) u_{0}^{+}, \varphi^{\prime}\left(s u_{0}^{-}\right) u_{0}^{-}\right\rangle$, and $\varphi_{1}(s, t)=\left\langle\frac{1}{s} \varphi^{\prime}\left(h^{+}(s, t)\right), \frac{1}{t} \varphi^{\prime}\left(h^{-}(s, t)\right)\right\rangle$. Note that

$$
\begin{aligned}
& \left\langle\varphi^{\prime}\left(t u_{0}^{ \pm}\right), u_{0}^{ \pm}\right\rangle>0 \quad \text { if } 0<t<1, \\
& \left\langle\varphi^{\prime}\left(t u_{0}^{ \pm}\right), u_{0}^{ \pm}\right\rangle<0 \quad \text { if } t>1 .
\end{aligned}
$$

Hence we have that $\operatorname{deg}\left(\varphi_{0}, D, 0\right)=1$. On the other hand, using (27) and property (a) of $\eta$, we have that $g=h$ on $\partial D$. Hence $\varphi_{1}=\varphi_{0}$ on $\partial D$ and $\operatorname{deg}\left(\varphi_{1}, D, 0\right)=\operatorname{deg}\left(\varphi_{0}, D, 0\right)=1$. This show that $\varphi_{1}(s, t)=0$ for some $(s, t) \in D$, and so $\eta(1, g(s, t))=h(s, t) \in \mathbb{M}_{0}$. Therefore $u_{0}$ is a critical point of $\varphi$.

\section{Sign-changing solutions}

For any $\lambda>0$, let $f_{\lambda}(x, t)=f(x, t)+\lambda r|t|^{r-2} t$ and

$$
\varphi_{\lambda}(u)=\varphi(u)-\lambda|u|_{r}^{r}, \quad u \in E
$$

Similarly, we define

$$
\begin{aligned}
& \mathbb{M}_{\lambda}:=\left\{u \in E: u^{ \pm} \neq 0,\left\langle\varphi_{\lambda}^{\prime}(u), u^{+}\right\rangle=\left\langle\varphi_{\lambda}^{\prime}(u), u^{-}\right\rangle=0\right\} \\
& \mathbb{N}_{\lambda}:=\left\{u \in E: u \neq 0,\left\langle\varphi_{\lambda}^{\prime}(u), u\right\rangle=0\right\}
\end{aligned}
$$

and

$$
m_{\lambda}:=\inf _{u \in \mathbb{M}_{\lambda}} \varphi_{\lambda}(u), \quad n_{\lambda}:=\inf _{u \in \mathbb{N}_{\lambda}} \varphi_{\lambda}(u)
$$

Lemma 4.1 Assume that $\left(h_{1}\right)-\left(h_{4}\right)$ hold. Then there exists a constant $\alpha>0$, which does not depend on $\lambda \in(0,1]$, such that

$$
\varphi_{\lambda}(u) \geq \alpha, \quad u \in \mathbb{N}_{\lambda}, \lambda \in(0,1]
$$

Proof For any $\varepsilon>0$, by $\left(h_{1}\right),\left(h_{2}\right)$, and Propositions 2.1 and 2.2 , for any $\lambda \in(0,1]$ and $u \in \mathbb{N}_{\lambda}$, we have

$$
\begin{aligned}
& \varepsilon c_{p}^{p}\|u\|^{p}+\left(C_{\varepsilon}+1\right) c_{r}^{r}\|u\|^{r} \\
& \quad \geq \varepsilon|u|_{p}^{p}+\left(C_{\varepsilon}+1\right)|u|_{r}^{r} \\
& \quad \geq \int_{\Omega} f_{\lambda}(x, u) u d x \\
& \quad=\int_{\Omega}\left(|\nabla u|^{p}+a(x)|\nabla u|^{q}\right) d x \\
& \quad \geq \begin{cases}\|u\|^{q} & \text { if }\|u\|<1, \\
\|u\|^{p} & \text { if }\|u\|>1 .\end{cases}
\end{aligned}
$$


Thus for any $u \in \mathbb{N}_{\lambda}$ with $\|u\|<1$, we have that

$$
\frac{1}{2}\|u\|^{q} \leq\left(C_{\varepsilon}+1\right) c_{r}^{r}\|u\|^{r},
$$

which implies that

$$
\|u\| \geq\left(\frac{1}{2\left(C_{\varepsilon}+1\right) c_{r}^{r}}\right)^{\frac{1}{r-q}}
$$

The proof is completed.

Now we are ready to prove Theorem 1.1.

Proof of Theorem 1.1 Clearly, for every $\lambda>0, f_{\lambda}$ satisfies conditions $\left(h_{1}\right)-\left(h_{3}\right)$ and $\left(h_{4}\right)^{\prime}$, and Lemmas 3.6 and 3.7 imply that there exists $u_{\lambda} \in \mathbb{M}_{\lambda}$ such that

$$
\varphi_{\lambda}\left(u_{\lambda}\right)=m_{\lambda} \quad \text { and } \quad \varphi_{\lambda}^{\prime}\left(u_{\lambda}\right)=0
$$

Furthermore, under assumptions $\left(h_{1}\right)-\left(h_{3}\right)$, we easily obtain that $\mathbb{M}_{0} \neq \emptyset$. Let $v_{0} \in \mathbb{M}_{0}$. Then $\varphi\left(v_{0}\right):=\kappa>0$ and $\left\langle\varphi^{\prime}\left(v_{0}\right), v_{0}^{ \pm}\right\rangle=0$. Therefore by Lemma 3.4 there exist $s_{\lambda}>0$ and $t_{\lambda}>0$ such that $s_{\lambda} v_{0}^{+}+t_{\lambda} v_{0}^{-} \in \mathbb{M}_{\lambda}$. Then from Corollary 3.2 and Lemma 4.1 we have

$$
\begin{aligned}
\kappa & =\varphi\left(v_{0}\right) \\
& \geq \varphi\left(s_{\lambda} v_{0}^{+}+t_{\lambda} v_{0}^{-}\right) \\
& \geq \varphi_{\lambda}\left(s_{\lambda} v_{0}^{+}+t_{\lambda} v_{0}^{-}\right) \\
& \geq m_{\lambda} \geq c_{*}, \quad \lambda \in(0,1) .
\end{aligned}
$$

Hence, we can choose a sequence $\left\{\lambda_{n}\right\}$ such that $\lambda_{n} \rightarrow 0$ as $n \rightarrow+\infty$ and

$$
u_{\lambda_{n}} \in \mathbb{M}_{\lambda_{n}}, \quad \varphi_{\lambda_{n}}\left(u_{\lambda_{n}}\right)=m_{\lambda_{n}} \rightarrow \bar{m}, \quad \varphi_{\lambda_{n}}^{\prime}\left(u_{\lambda_{n}}\right)=0
$$

Thus we only need to prove the following claims to complete the proof of Theorem 1.1.

Claim $1\left\{u_{\lambda_{n}}\right\}$ is bounded in E.

Arguing by contradiction, suppose that $\left\|u_{\lambda_{n}}\right\| \rightarrow+\infty$ as $n \rightarrow+\infty$. We define the sequence $v_{n}=\frac{u_{\lambda_{n}}}{\left\|u_{\lambda_{n}}\right\|}, n=1,2, \ldots$ It is clear that $\left\{v_{n}\right\} \subset E$ and $\left\|v_{n}\right\|=1$ for any $n \in N$. Therefore, going if necessary to a subsequence, we may assume that

$$
\begin{aligned}
& v_{n} \rightarrow v \quad \text { in } E, \\
& v_{n} \rightarrow v \quad \text { in } L^{\vartheta}(\Omega), 1 \leq \vartheta<p^{*}, \\
& v_{n}(x) \rightarrow v(x) \quad \text { a.e. on } \Omega .
\end{aligned}
$$


If $v=0$, then $v_{n} \rightarrow 0$ in $L^{\vartheta}(\Omega)$ for $1 \leq \vartheta<p^{*}$. Fix $R>\left[q\left(m_{0}+1\right)\right]^{\frac{1}{p}}$. Using conditions $\left(h_{1}\right)-$ $\left(h_{2}\right)$ and the Lebesgue dominated convergence theorem, we deduce that

$$
\limsup _{n \rightarrow \infty} \int_{\Omega} F\left(x, R v_{n}\right) d x \leq R^{p} \lim _{n \rightarrow \infty}\left(\left|v_{n}\right|_{p}^{p}+C_{3} R^{r}\left|v_{n}\right|_{r}^{r}\right)=0
$$

for some constant $C_{3}>0$.

Let $t_{n}=\frac{R}{\left\|u_{n}\right\|}$. Then by (32) and Corollary 3.3 we get that

$$
\begin{aligned}
m_{\lambda_{n}}= & \varphi_{\lambda_{n}}\left(u_{\lambda_{n}}\right) \geq \varphi_{\lambda_{n}}\left(t_{n} u_{\lambda_{n}}\right)=\varphi_{\lambda_{n}}\left(R v_{n}\right) \\
= & \int_{\Omega}\left(\frac{1}{p} R^{p}\left|\nabla v_{n}\right|^{p}+\frac{a(x)}{q} R^{q}\left|\nabla v_{n}\right|^{q}\right) d x \\
& -\int_{\Omega}\left(F\left(x, R v_{n}\right)+\lambda_{n} R^{r}\left|v_{n}\right|^{r}\right) d x \\
\geq & \frac{1}{q} R^{p}-\int_{\Omega}\left(F\left(x, R v_{n}\right)+\lambda_{n} R^{r}\left|v_{n}\right|^{r}\right) d x \\
= & \frac{1}{q} R^{p}+o(1)>m_{0}+1+o(1),
\end{aligned}
$$

which yields a contradiction. Thus $v \neq 0$.

By $\left(h_{3}\right)$ we get

$$
\lim _{k \rightarrow+\infty} \frac{F\left(x, u_{\lambda_{n}}(x)\right)}{\left\|u_{\lambda_{n}}\right\|^{q}}=\lim _{k \rightarrow+\infty} \frac{F\left(x, u_{\lambda_{n}}(x)\right)}{\left|u_{\lambda_{n}}(x)\right|^{q}}\left|v_{n}(x)\right|^{q}=+\infty
$$

for all $x \in \Omega_{0}:=\{x \in \Omega: v(x) \neq 0\}$. Therefore, using (21), (30), and Fatou's lemma, we have

$$
\begin{aligned}
0 \leq & \lim _{n \rightarrow \infty} \frac{\varphi_{\lambda_{n}}\left(u_{\lambda_{n}}\right)}{\left\|u_{\lambda_{n}}\right\|^{q}} \\
\leq & \lim _{n \rightarrow \infty}\left[\frac{1}{p} \frac{\int_{\Omega}\left(\left|\nabla u_{\lambda_{n}}\right|^{p}+a(x)\left|\nabla u_{\lambda_{n}}\right|^{q}\right) d x}{\left\|u_{n}\right\|^{q}}\right. \\
& \left.-\int_{\Omega} \frac{F\left(x, u_{\lambda_{n}}\right)+\lambda_{n}\left|u_{\lambda_{n}}\right|^{r}}{\left\|u_{\lambda_{n}}\right\|^{q}} d x\right] \\
\leq & \lim _{n \rightarrow \infty}\left[\frac{1}{p} \frac{\int_{\Omega}\left(\left|\nabla u_{\lambda_{n}}\right|^{p}+a(x)\left|\nabla u_{\lambda_{n}}\right|^{q}\right) d x}{\left\|u_{\lambda_{n}}\right\|^{q}}-\int_{\Omega} \frac{F\left(x, u_{\lambda_{n}}\right)}{\left\|u_{\lambda_{n}}\right\|^{q}} d x\right] \\
\leq & \frac{1}{p}-\lim _{n \rightarrow \infty} \int_{\Omega} \frac{F\left(x, u_{\lambda_{n}}\right)}{\left\|u_{\lambda_{n}}\right\|^{q}} d x \\
= & \frac{1}{p}-\lim _{n \rightarrow \infty} \int_{\Omega} \frac{F\left(x, u_{\lambda_{n}}\right)-C_{2}}{\left\|u_{\lambda_{n}}\right\|^{q}} d x \\
\leq & \frac{1}{p}-\liminf _{n \rightarrow \infty} \int_{\Omega_{0}} \frac{F\left(x, u_{\lambda_{n}}\right)-C_{2}}{\left\|u_{\lambda_{n}}\right\|^{q}} d x \\
= & \frac{1}{p}-\liminf _{n \rightarrow \infty} \int_{\Omega_{0}} \frac{F\left(x, u_{\lambda_{n}}(x)\right)}{\left|u_{\lambda_{n}}(x)\right|^{q}}\left|v_{n}(x)\right|^{q} d x \\
\rightarrow & -\infty,
\end{aligned}
$$


which is contradiction. The proof of Claim 1 is complete. Thus there exist a subsequence of $\left\{\lambda_{n}\right\}$, still denoted by $\left\{\lambda_{n}\right\}$, and $u_{0} \in E$ such that

$$
u_{\lambda_{n}} \rightarrow u_{0} \quad \text { in } E .
$$

Claim $2 \varphi\left(u_{0}\right)=m_{0}$ and $\varphi^{\prime}\left(u_{0}\right)=0$.

By the Sobolev embedding theorem, $u_{\lambda_{n}} \rightarrow u_{0}$ in $L^{\vartheta}(\Omega), 1 \leq \vartheta<p^{*}$, and $u_{\lambda_{n}}(x) \rightarrow u_{0}(x)$ a.e. on $\Omega$. By $\left(h_{2}\right)$ and the Hölder inequality it is easy to directly compute that

$$
\begin{aligned}
\int_{\Omega}\left|f\left(x, u_{\lambda_{n}}\right)\right|\left|u_{n}-u_{0}\right| d x \\
\leq \int_{\Omega} C\left(1+\left|u_{\lambda_{n}}\right|^{r-1}\right)\left|u_{n}-u_{0}\right| d x \\
\leq C \int_{\Omega}\left|u_{n}\right|^{r-1}\left|u_{\lambda_{n}}-u_{0}\right| d x+C \int_{\Omega}\left|u_{n}-u_{0}\right| d x \\
\leq C\left(\int_{\Omega}\left|u_{\lambda_{n}}\right|^{(r-1) r^{\prime}} d x\right)^{\frac{1}{r}}\left(\int_{\Omega}\left|u_{\lambda_{n}}-u_{0}\right|^{r} d x\right)^{\frac{1}{r}} \\
\quad+C \int_{\Omega}\left|u_{\lambda_{n}}-u_{0}\right| d x \\
=C\left(\int_{\Omega}\left|u_{\lambda_{n}}\right|^{r} d x\right)^{\frac{r-1}{r}}\left(\int_{\Omega}\left|u_{\lambda_{n}}-u_{0}\right|^{r} d x\right)^{\frac{1}{r}}+C \int_{\Omega}\left|u_{\lambda_{n}}-u_{0}\right| d x \\
=C\left|u_{\lambda_{n}}\right|_{r}^{r-1}\left|u_{\lambda_{n}}-u_{0}\right|_{r}+C\left|u_{\lambda_{n}}-u_{0}\right|_{1} \\
\rightarrow 0 \quad \text { as } n \rightarrow \infty
\end{aligned}
$$

where $\frac{1}{r}+\frac{1}{r^{\prime}}=1$. Then, using (30), (34), and $\left(h_{2}\right)$, we deduce

$$
\begin{aligned}
\left\langle L\left(u_{\lambda_{n}}\right)-L\left(u_{0}\right), u_{\lambda_{n}}-u_{0}\right\rangle= & \left\langle\varphi_{\lambda_{n}}^{\prime}\left(u_{\lambda_{n}}\right)-\varphi^{\prime}\left(u_{0}\right), u_{\lambda_{n}}-u_{0}\right\rangle \\
& +\int_{\Omega}\left[f\left(x, u_{\lambda_{n}}\right)+\lambda_{n} r\left|u_{\lambda_{n}}\right|^{r-2} u_{\lambda_{n}}\right]\left(u_{\lambda_{n}}-u_{0}\right) d x \\
& -\int_{\Omega} f\left(x, u_{0}\right)\left(u_{\lambda_{n}}-u_{0}\right) d x \\
\rightarrow & 0 \quad \text { as } n \rightarrow+\infty .
\end{aligned}
$$

Since $L$ is of type $(S)_{+}$, we see that

$$
u_{\lambda_{n}} \rightarrow u_{0} \quad \text { in } E
$$

and so $u_{\lambda_{n}}^{ \pm} \rightarrow u_{0}^{ \pm}$in $E$. Thus from (30) it follows that $\varphi\left(u_{0}\right)=\bar{m}$.

Moreover, by Proposition 2.3, (30), and (35) we get

$$
\begin{aligned}
\left\langle\varphi^{\prime}\left(u_{0}\right), \eta\right\rangle & =\left\langle L\left(u_{0}\right), \eta\right\rangle-\int_{\Omega} f\left(x, u_{0}\right) \eta d x \\
& =\lim _{n \rightarrow+\infty}\left(\left\langle L\left(u_{\lambda_{n}}\right), \eta\right\rangle-\int_{\Omega}\left[f\left(x, u_{\lambda_{n}}\right)+\lambda_{n} r\left|u_{\lambda_{n}}\right|^{r-2} u_{\lambda_{n}}\right] \eta d x\right)
\end{aligned}
$$




$$
\begin{aligned}
& =\lim _{n \rightarrow+\infty}\left\langle\varphi_{\lambda_{n}}^{\prime}\left(u_{\lambda_{n}}\right), \eta\right\rangle \\
& =0, \quad \eta \in E .
\end{aligned}
$$

This shows that $\varphi^{\prime}\left(u_{0}\right)=0$. Again from Lemma 4.1 and (35) we have

$$
\begin{aligned}
\int_{\Omega} & {\left[\frac{1}{q} f\left(x, u_{0}^{ \pm}\right) u_{0}^{ \pm}-F\left(x, u_{0}^{ \pm}\right)\right] d x+\int_{\Omega}\left(\frac{1}{p}-\frac{1}{q}\right)\left|\nabla u_{0}^{ \pm}\right|^{p} d x } \\
= & \lim _{n \rightarrow+\infty} \int_{\Omega}\left[\frac{1}{q} f\left(x, u_{\lambda_{n}}^{ \pm}\right) u_{\lambda_{n}}^{ \pm}-F\left(x, u_{\lambda_{n}}^{ \pm}\right)\right] d x \\
& +\lim _{n \rightarrow+\infty} \int_{\Omega}\left(\frac{1}{p}-\frac{1}{q}\right)\left|\nabla u_{\lambda_{n}}^{ \pm}\right|^{p} d x+\lim _{n \rightarrow+\infty} \frac{\lambda_{n}(r-q)}{q}\left|u_{\lambda_{n}}^{ \pm}\right|_{r}^{r} \\
= & \lim _{n \rightarrow+\infty}\left[\varphi_{\lambda_{n}}\left(u_{\lambda_{n}}^{ \pm}\right)-\frac{1}{q}\left\langle\varphi_{\lambda_{n}}^{\prime}\left(u_{\lambda_{n}}^{ \pm}\right), u_{n}^{ \pm}\right\rangle\right] \\
= & \lim _{n \rightarrow+\infty} \varphi_{\lambda_{n}}\left(u_{\lambda_{n}}^{ \pm}\right) \\
\geq & \alpha \alpha>0 .
\end{aligned}
$$

This, together with (6) $(t=0)$, shows that $u_{0}^{ \pm} \neq 0$. Therefore

$$
\varphi^{\prime}\left(u_{0}\right)=0, \quad u_{0} \in \mathbb{M}_{0}, \quad \text { and } \quad \varphi\left(u_{0}\right)=\bar{m} \geq m_{0} .
$$

Next, we will prove that $\varphi\left(u_{0}\right)=m_{0}$. Let $\varepsilon$ be any positive number. Since $m_{0}=\inf _{u \in \mathbb{M}_{0}} \varphi(u)$, there exists $v_{\varepsilon} \in \mathbb{M}_{0}$ such that $\varphi\left(v_{\varepsilon}\right)<m_{0}+\varepsilon$. Then $\left(h_{3}\right)$ implies that there exists $M_{\varepsilon}>1$ such that, for $s \geq M_{\varepsilon}$ or $t \geq M_{\varepsilon}$,

$$
\begin{aligned}
\varphi_{\lambda_{n}}\left(s v_{\varepsilon}^{+}+t v_{\varepsilon}^{-}\right)= & \int_{\Omega}\left(\frac{s^{p}}{p}\left|\nabla v_{\varepsilon}^{+}\right|^{p}+\frac{s^{q}}{q}\left|\nabla v_{\varepsilon}^{+}\right|^{q}\right) d x-\int_{\Omega} F\left(x, s v_{\varepsilon}^{+}\right) d x \\
& -\lambda_{n} s^{r} \int_{\Omega}\left|v_{\varepsilon}^{+}\right|^{r} d x \\
& +\int_{\Omega}\left(\frac{t^{p}}{p}\left|\nabla v_{\varepsilon}^{-}\right|^{p}+\frac{t^{q}}{q}\left|\nabla v_{\varepsilon}^{-}\right|^{q}\right) d x-\int_{\Omega} F\left(x, t v_{\varepsilon}^{-}\right) d x \\
& -\lambda_{n} t^{r} \int_{\Omega}\left|v_{\varepsilon}^{-}\right|^{r} d x \\
\leq & \int_{\Omega}\left(\frac{s^{p}}{p}\left|\nabla v_{\varepsilon}^{+}\right|^{p}+\frac{s^{q}}{q}\left|\nabla v_{\varepsilon}^{+}\right|^{q}\right) d x-\int_{\Omega} F\left(x, s v_{\varepsilon}^{+}\right) d x \\
& +\int_{\Omega}\left(\frac{t^{p}}{p}\left|\nabla v_{\varepsilon}^{-}\right|^{p}+\frac{t^{q}}{q}\left|\nabla v_{\varepsilon}^{-}\right|^{q}\right) d x-\int_{\Omega} F\left(x, t v_{\varepsilon}^{-}\right) d x \\
< & 0 .
\end{aligned}
$$

In view of Lemma 3.4, there exists a pair $\left(s_{n}, t_{n}\right)$ of positive numbers such that $s_{n} v_{\varepsilon}^{+}+$ $t_{n} v_{\varepsilon}^{-} \in \mathbb{M}_{\lambda_{n}}$, which, together with (38), implies $0<s_{n}, t_{n}<M_{\varepsilon}$. Thus from Lemma 3.1 and $\left\langle\varphi^{\prime}\left(v_{\varepsilon}\right), v_{\varepsilon}^{ \pm}\right\rangle=0$ we have

$$
\begin{aligned}
m_{0}+\varepsilon & >\varphi\left(v_{\varepsilon}\right)=\varphi_{\lambda_{n}}\left(v_{\varepsilon}\right)+\lambda_{n}\left|v_{\varepsilon}\right|_{r}^{r} \\
& \geq \varphi_{\lambda_{n}}\left(s_{n} v_{\varepsilon}^{+}+t_{n} v_{\varepsilon}^{-}\right)+\frac{1-s_{n}^{q}}{q}\left\langle\varphi_{\lambda_{n}}^{\prime}\left(v_{\varepsilon}\right), v_{\varepsilon}^{+}\right\rangle+\frac{1-t_{n}^{q}}{q}\left\langle\varphi_{\lambda_{n}}^{\prime}\left(v_{\varepsilon}\right), v_{\varepsilon}^{-}\right\rangle
\end{aligned}
$$




$$
\begin{aligned}
& +\int_{\Omega} g\left(s_{n}\right)\left|\nabla v_{\varepsilon}^{+}\right|^{p} d x+\int_{\Omega} g\left(t_{n}\right)\left|\nabla v_{\varepsilon}^{-}\right|^{p} d x \\
\geq & m_{\lambda_{n}}-\frac{1+K_{\varepsilon}^{q}}{q}\left|\left\langle\varphi_{\lambda_{n}}^{\prime}\left(v_{\varepsilon}\right), v_{\varepsilon}^{+}\right\rangle\right|-\frac{1+K_{\varepsilon}^{q}}{q}\left|\left\langle\varphi_{\lambda_{n}}^{\prime}\left(v_{\varepsilon}\right), v_{\varepsilon}^{-}\right\rangle\right| \\
= & m_{\lambda_{n}}-\frac{\left(1+K_{\varepsilon}^{q}\right) r \lambda_{n}}{q}\left|v_{\varepsilon}^{+}\right|_{r}^{r}-\frac{\left(1+K_{\varepsilon}^{q}\right) r \lambda_{n}}{q}\left|v_{\varepsilon}^{-}\right|_{r}^{r},
\end{aligned}
$$

which yields

$$
\bar{m}=\lim _{n \rightarrow+\infty} m_{\lambda_{n}} \leq m_{0}+\varepsilon .
$$

Since $\varepsilon>0$ is arbitrary, we have $\bar{m} \leq m_{0}$. Thus $\bar{m}=m_{0}$, that is, $\varphi\left(u_{0}\right)=m_{0}$.

Now we show that $u_{0}$ has exactly two nodal domains. Let $u_{0}=u_{1}+u_{2}+u_{3}$, where

$$
\begin{aligned}
& u_{1} \geq 0, \quad u_{2} \leq 0, \quad \Omega_{1} \cap \Omega_{2}=\emptyset, \\
& \left.u_{1}\right|_{\Omega \backslash\left(\Omega_{1} \cup \Omega_{2}\right)}=\left.u_{2}\right|_{\Omega \backslash\left(\Omega_{1} \cup \Omega_{2}\right)}=\left.u_{3}\right|_{\Omega_{1} \cup \Omega_{2}}, \\
& \Omega_{1}:=\left\{x \in \Omega \mid u_{1}(x)>0\right\}, \quad \Omega_{2}:=\left\{x \in \Omega \mid u_{1}(x)<0\right\},
\end{aligned}
$$

and $\Omega_{i}(i=1,2)$ are connected open subsets of $\Omega$.

Setting $v=u_{1}+u_{2}$, we see that $v^{+}=u_{1}$ and $v^{-}=u_{2}$, that is, $v^{ \pm} \neq 0$. Noting that $\varphi^{\prime}\left(u_{0}\right)=0$, by a simple computation we have

$$
\left\langle\varphi^{\prime}(v), v^{+}\right\rangle=\left\langle\varphi^{\prime}(v), v^{-}\right\rangle=0
$$

By Lemma 3.1 and again by (40) and (41) we conclude that

$$
\begin{aligned}
m_{0}= & \varphi\left(u_{0}\right)=\varphi\left(u_{0}\right)-\frac{1}{q}\left\langle\varphi^{\prime}\left(u_{0}\right), u_{0}\right\rangle \\
= & \varphi(v)+\varphi\left(u_{3}\right)-\frac{1}{q}\left(\left\langle\varphi^{\prime}(v), v\right\rangle+\left\langle\varphi^{\prime}\left(u_{3}\right), u_{3}\right\rangle\right) \\
\geq & \sup _{s, t \geq 0}\left[\varphi\left(s v^{+}+t v^{-}\right)+\frac{1-s^{q}}{q}\left\langle\varphi^{\prime}(v), v^{+}\right\rangle+\frac{1-t^{q}}{q}\left\langle\varphi^{\prime}(v), v^{-}\right\rangle\right. \\
& \left.+\int_{\Omega} g(s)\left|\nabla v^{+}\right|^{p} d x+\int_{\Omega} g(t)\left|\nabla v^{-}\right|^{p} d x\right]+\varphi\left(u_{3}\right)-\frac{1}{q}\left\langle\varphi^{\prime}\left(u_{3}\right), u_{3}\right\rangle \\
\geq & \sup _{s, t \geq 0} \varphi\left(s v^{+}+t v^{-}\right)+\varphi\left(u_{3}\right)-\frac{1}{q}\left\langle\varphi^{\prime}\left(u_{3}\right), u_{3}\right\rangle \\
\geq & m_{0}+\int_{\Omega}\left(\frac{1}{q} f\left(x, u_{3}\right) u_{3}-F\left(x, u_{3}\right)\right) d x+\left(\frac{1}{p}-\frac{1}{q}\right) \int_{\Omega}\left|\nabla u_{3}\right|^{p} d x \\
\geq & m_{0}+\int_{\Omega}\left(\frac{1}{q} f\left(x, u_{3}\right) u_{3}-F\left(x, u_{3}\right)\right) d x,
\end{aligned}
$$

which, together with (3), shows that $u_{3}=0$. Therefore $u_{0}$ has exactly two nodal domains.

Proof of Theorem 1.2 By Theorem 1.1 there exists $u_{0} \in \mathbb{M}_{0}$ such that $\varphi\left(u_{0}\right)=m_{0}$. Since $u_{0}^{ \pm} \in \mathbb{N}_{0}$, we have $m_{0}=\varphi\left(u_{0}\right)=\varphi\left(u_{0}^{+}\right)+\varphi\left(u_{0}^{-}\right) \geq 2 n_{0}$. 


\section{Acknowledgements}

The authors would like to thank the referees for their useful suggestions.

\section{Funding}

This work is supported by the Fundamental Research Funds for the Central Universities (No. 3072019CF2402), the National Natural Science Foundation of China (No. 11201095), the Postdoctoral research startup foundation of Heilongjiang (No. LBH-Q14044), and the Science Research Funds for Overseas Returned Chinese Scholars of Heilongjiang Province (No. LC201502).

\section{Abbreviations}

Not applicable.

\section{Availability of data and materials}

Not applicable.

\section{Competing interests}

The authors declare that they have no competing interests.

\section{Authors' contributions}

The authors declare that the study was realized in collaboration with equal responsibility. All authors read and approved the final manuscript.

\section{Author details}

${ }^{1}$ College of Aerospace and Civil Engineering, Harbin Engineering University, Harbin, P.R. China. ${ }^{2}$ School of Mathematical Sciences, Harbin Engineering University, Harbin, P.R. China. ${ }^{3}$ College of Automation, Harbin Engineering University, Harbin, P.R. China.

\section{Publisher's Note}

Springer Nature remains neutral with regard to jurisdictional claims in published maps and institutional affiliations.

Received: 27 September 2019 Accepted: 27 January 2020 Published online: 03 February 2020

\section{References}

1. Liu, W.L., Dai, G.W.: Existence and multiplicity results for double phase problem. J. Differ. Equ. 265(9), 4311-4334 (2018)

2. Zhikov, V.V:: Averaging of functionals of the calculus of variations and elasticity theory. Izv. Akad. Nauk SSSR, Ser. Mat. 50(4), 675-710 (1986)

3. Zhikov, V.V.: On Lavrentiev's phenomenon. Russ. J. Math. Phys. 3(2), 249-269 (1995)

4. Zhikov, V.V.: On some variational problems. Russ. J. Math. Phys. 5(1), 105-116 (1997)

5. Zhikov, V.V., Kozlov, S.M., Oleinik, O.A.: Homogenization of Differential Operators and Integral Functionals. Springer, Berlin (1994)

6. Perera, K., Squassina, M.: Existence results for double-phase problems via Morse theory. Commun. Contemp. Math. 20(2), 1-14 (2018)

7. Papageorgiou, N.S., Radulescu, V.D., Repovs, D.D.: Double-phase problems with reaction of arbitrary growth. Z. Angew. Math. Phys. 69(4), 1-21 (2018)

8. Cencelj, M., Radulescu, V.D., Repovs, D.D.: Double phase problems with variable growth. Nonlinear Anal., Theory Methods Appl. 177(A), 270-287 (2018)

9. Liu, W.L., Dai, G.W.: Three ground state solutions for double phase problem. J. Math. Phys. 59(12), 1-7 (2018)

10. Colasuonno, F., Squassina, M.: Eigenvalues for double phase variational integrals. Ann. Mat. Pura Appl. 195(6), 1917-1959 (2016)

11. Mao, A.M., Zhang, Z.T.: Sign-changing and multiple solutions of Kirchhoff type problems without the p.s. condition. Nonlinear Anal., Theory Methods Appl. 70(3), 1275-1287 (2009)

12. Musielak, J.: Orlicz Spaces and Modular Spaces. Springer, Berlin (1983)

13. Benkirane, A., Sidi El Vally, M.: Variational inequalities in Musielak-Orlicz-Sobolev spaces. Bull. Belg. Math. Soc. 21(5), 787-811 (2014)

14. Fan, X., Guan, C.X.: Uniform convexity of Musielak-Oorlicz-Sobolev spaces and applications. Nonlinear Anal., Theory Methods Appl. 73(1), 163-175 (2010)

15. Chang, K.C.: Critical Point Theory and Applications. Shanghai Sci. Technol., Shanghai (1996)

16. Miranda, C.: Un'osservazione su un teorema di Brouwer. Boll. Unione Mat. Ital. 3, 5-7 (1940)

17. Willem, M.: Minimax Theorems. Birkhäuser, Basel (1996) 